\title{
Latest results from NA62
}

\author{
Alina Kleimenova*† \\ UCL, Louvain-la-Neuve \\ E-mail: alina.kleimenova@cern.ch
}

\begin{abstract}
The ultra rare kaon decay $K \rightarrow \pi v \bar{v}$, being one of the theoretically cleanest meson decays, is very sensitive to the effects of New Physics at high mass scales. NA62 is a fixed-target experiment at the CERN SPS designed to measure the branching ratio of the $K^{+} \rightarrow \pi^{+} \nu \bar{v}$ decay with $10 \%$ precision using a novel decay-in-flight technique. NA62 took data during run periods in 2016, 2017 and 2018. The results from the analysis of the data collected by NA62 in 2016, corresponding to $\left(1.21 \pm 0.04_{\text {syst }}\right) \times 10^{11} K^{+}$decays ( $\%$ of full available statistics), will be presented and future prospects will be reviewed. Although NA62 was designed to measure $K \rightarrow \pi \nu \bar{v}$, it's also sensitive to other rare and forbidden kaon decays, especially those with two leptons in the final state. The status and prospects of searches for lepton flavour and lepton number violation in kaon decays at the NA62 experiment will also be discussed.
\end{abstract}

XXVII International Workshop on Deep-Inelastic Scattering and Related Subjects - DIS2019 8-12 April, 2019

Torino, Italy

* Speaker.

${ }^{\dagger}$ On behalf of the NA62 Collaboration: R. Aliberti, F. Ambrosino, R. Ammendola, B. Angelucci, A. Antonelli, G. Anzivino, R. Arcidiacono, T. Bache, M. Barbanera, J. Bernhard, A. Biagioni, L. Bician, C. Biino, A. Bizzeti, T. Blazek, B. Bloch-Devaux, V. Bonaiuto, M. Boretto, M. Bragadireanu, D. Britton, F. Brizioli, M.B. Brunetti, D. Bryman, F. Bucci, T. Capussela, J. Carmignani, A. Ceccucci, P. Cenci, V. Cerny, C. Cerri, B. Checcucci, A. Conovaloff, P. Cooper, E. Cortina Gil, M. Corvino, F. Costantini, A. Cotta Ramusino, D. Coward, G. D’Agostini, J. Dainton, P. Dalpiaz, H. Danielsson, N. De Simone, D. Di Filippo, L. Di Lella, N. Doble, B. Dobrich, F. Duval, V. Duk, J. Engelfried, T. Enik, N. Estrada-Tristan, V. Falaleev, R. Fantechi, V. Fascianelli, L. Federici, S. Fedotov, A. Filippi, M. Fiorini, J. Fry, J. Fu, A. Fucci, L. Fulton, E. Gamberini, L. Gatignon, G. Georgiev, S. Ghinescu, A. Gianoli, M. Giorgi, S. Giudici, F. Gonnella, E. Goudzovski, C. Graham, R. Guida, E. Gushchin, F. Hahn, H. Heath, E.B. Holzer, T. Husek, O. Hutanu, D. Hutchcroft, L. Iacobuzio, E. Iacopini, E. Imbergamo, B. Jenninger, J. Jerhot, R.W. Jones, K. Kampf, V. Kekelidze, S. Kholodenko, G. Khoriauli, A. Khotyantsev, A. Kleimenova, A. Korotkova, M. Koval, V. Kozhuharov, Z. Kucerova, Y. Kudenko, J. Kunze, V. Kurochka, V. Kurshetsov, G. Lanfranchi, G. Lamanna, E. Lari, G. Latino, P. Laycock, C. Lazzeroni, M. Lenti, G. Lehmann Miotto, E. Leonardi, P. Lichard, L. Litov, R. Lollini, D. Lomidze, A. Lonardo, P. Lubrano, M. Lupi, N. Lurkin, D. Madigozhin, I. Mannelli, G. Mannocchi, A. Mapelli, F. Marchetto, R. Marchevski, S. Martellotti, P. Massarotti, K. Massri, E. Maurice, M. Medvedeva, A. Mefodev, E. Menichetti, E. Migliore, E. Minucci, M. Mirra, M. Misheva, N. Molokanova, M. Moulson, S. Movchan, M. Napolitano, I. Neri, F. Newson, A. Norton, M. Noy, T. Numao, V. Obraztsov, A. Ostankov, S. Padolski, R. Page, V. Palladino, A. Parenti, C. Parkinson, E. Pedreschi, M. Pepe, M. Perrin-Terrin, L. Peruzzo, P. Petrov, Y. Petrov, F. Petrucci, R. Piandani, M. Piccini, J. Pinzino, I. Polenkevich, L. Pontisso, Yu. Potrebenikov, D. Protopopescu, M. Raggi, A. Romano, P. Rubin, G. Ruggiero, V. Ryjov, A. Salamon, C. Santoni, G. Saracino, F. Sargeni, S. Schuchmann, V. Semenov, A. Sergi, A. Shaikhiev, S. Shkarovskiy, D. Soldi, V. Sugonyaev, M. Sozzi, T. Spadaro, F. Spinella, A. Sturgess, J. Swallow, S. Trilov, P. Valente, B. Velghe, S. Venditti, P. Vicini, R. Volpe, M. Vormstein, H. Wahl, R. Wanke, B. Wrona, O. Yushchenko, M. Zamkovsky, A. Zinchenko. 


\section{Introduction}

The $K \rightarrow \pi v \bar{v}$ is a flavour changing neutral current process that proceeds through the box and penguin diagrams that are dominated by top-quark exchange. This process is highly suppressed due to the quadratic GIM mechanism and the small value of CKM element $\left|V_{t d}\right|$. The Standard Model (SM) prediction for this decay is $\mathscr{B}\left(K^{+} \rightarrow \pi^{+} v \bar{v}\right)=(8.4 \pm 1.0) \times 10^{-11}$ [1], where the main source of theoretical uncertainties is the experimental values of CKM parameters.

The $K \rightarrow \pi v \bar{v}$ decay is sensitive to physics beyond the SM. In particular to such extensions as Randall-Sundrum models with protective custodial symmetry [2], Minimal Supersymmetric extension of the Standard Model [3, 4], simplified Z and Z' [5], littlest Higgs with T-Parity [6] and LFU violation models [7].

The latest experimental result on the branching ratio measurement is $\mathscr{B}\left(K^{+} \rightarrow \pi^{+} v \bar{v}\right)=$ $\left(17.3_{-10.5}^{+11.5}\right) \times 10^{-11}$. It was obtained by experiments E787 and E949 at BNL, which collected a total of 7 events using decay-at-rest technique [8]. This result is consistent with the SM, but still far from the precision of the SM prediction.

\section{The NA62 experiment}

NA62 is a fixed target experiment located in the North Area of CERN which aims to measure $\mathscr{B}\left(K^{+} \rightarrow \pi^{+} v \bar{v}\right)$ with $10 \%$ precision using a decay-in-flight technique. In order to achieve this goal a total of $10^{13}$ kaon decays must be collected using $400 \mathrm{GeV} / \mathrm{c}$ protons from SPS. The protons impinge on a beryllium target and produce a secondary hadron beam composed of $70 \%$ pions, $23 \%$ protons and $6 \%$ kaons at nominal momentum $75 \mathrm{GeV} / \mathrm{c}$ and $1 \%$ rms momentum bite.

The signal consists of one incoming kaon track and one outgoing pion track, with no other activity in the detector. The main background contribution comes from the kaon decay channels with the highest branching ratio: mostly $K^{+} \rightarrow \mu^{+} v(\gamma)$ and $K^{+} \rightarrow \pi^{+} \pi^{0}(\gamma)$. There are also contributions from upstream decays in the beam line and interactions between the beam particles and upstream detectors. The high background rejection required is provided by combining various techniques: kinematic suppression, highghly efficient vetos and time resolution.

The NA62 beam line and detector layout are described in detail in [9] and are shown in Figure 1. Particle identification and time measurements of the incoming beam kaons are provided by a differential Cherenkov counter (KTAG). It is followed by a three silicon pixel stations (GTK), which form a beam spectrometer to measure momentum, direction and time of beam particles. In order to veto inelastic scattering occurring in the GTK stations scintillating anti-counters (CHANTI) are installed around the beam pipe after the last station of the beam spectrometer. Momentum and direction of downstream particles are measured by the STRAW spectrometer placed in the end of $110 \mathrm{~m}$ long vacuum tank. Particle identification in the momentum range $15-35 \mathrm{GeV} / \mathrm{c}$ for downstream particles is carried out by the Ring Imaging Cherenkov detector (RICH). Further particle identification is provided by two hadronic calorimeters and muon veto detectors (MUV1,2,3) placed at the end of the beam line. Twelve ring shaped Large Angle Vetoes (LAV), with increasing diameter with distance from the target, surround the vacuum tube. Along with the Liquid Krypton calorimeter (LKr), the Inner Ring Calorimeter (IRC) and the Small Angle Calorimeter (SAC) LAV provides hermetic coverage for photons emitted in kaon decays with polar angles up to $50 \mathrm{mrad}$. 


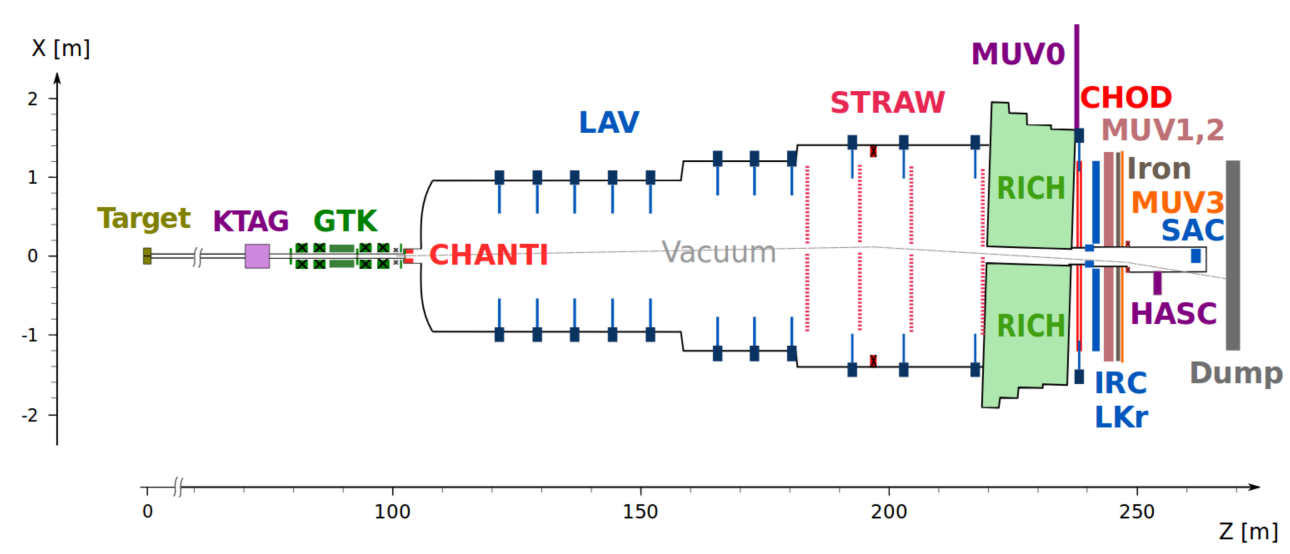

Figure 1: Schematic top view of the NA62 experiment

Additional counters (MUV0, HASC) installed at optimized locations provide hermetic coverage for charged particles produced in multi-track kaon decays.

\section{The $K^{+} \rightarrow \pi^{+} v \bar{v}$ analysis}

The $K^{+} \rightarrow \pi^{+} v \bar{v}$ signature, as it was stated above, is one track in the initial and final state. The main kinematic variable is the squared missing mass $m_{m i s s}^{2}=\left(P_{K}-P_{\pi}\right)^{2}$, where $P_{K}$ and $P_{\pi}$ are the 4-momenta of the $K^{+}$and $\pi^{+}$respectively. The analysis is done in the $\pi^{+}$momentum range between 15 and $35 \mathrm{GeV} / \mathrm{c}$. This condition improves the rejection of $K^{+} \rightarrow \pi^{+} \pi^{0}$ decay by ensuring that at least $40 \mathrm{GeV}$ of electromagnetic energy is deposited in the calorimeters and optimizing the $\pi^{+} / \mu^{+}$identification with the RICH.

Events with a single track topology are selected using the STRAW, CHOD and RICH. The track reconstructed with STRAW must have matching signals in CHOD and a reconstructed ring in the RICH. An upstream kaon track reconstructed with GTK and identified with KTAG is then matched in time with the downstream track. The kaon decay vertex is built from the intersection of the two tracks and is required to be within a $50 \mathrm{~m}$ long decay region starting downstream of the last GTK station. Then downstream track is identified by using the combination of the RICH measurement and a multivariate analysis of calorimetric information providing $10^{8}$ muon suppression for $64 \%$ pion efficiency. The remaining background is mostly represented by $K^{+} \rightarrow \pi^{+} \pi^{0}$, which is further suppressed using the electromagnetic information (LKr, SAC, IRC and LAV). Events with extra energy deposits in time with the pion track are rejected, providing a $\pi^{0}$ suppression of $3 \times 10^{-8}$.

The analysis is done in two separate regions defined in the $m_{m i s s}^{2}$ variable below and above the $K \rightarrow \pi^{+} \pi^{0}$ peak as $(0,0.01) \mathrm{GeV}^{2} / \mathrm{c}^{4}$ (Region 1 - R1) and $(0.026,0.068) \mathrm{GeV}^{2} / \mathrm{c}^{4}$ (Region 2 $\mathrm{R} 2$ ). The choice of the boundaries was driven by the resolution of the squared missing mass, which is $10^{-3} \mathrm{GeV}^{2} / \mathrm{c}^{4}$. To protect against track momenta mis-reconstruction, $m_{m i s s}^{2}$ is computed in three different ways: using STRAW for $p_{\pi^{+}}$and GTK for $p_{K^{+}}$, RICH for $p_{\pi^{+}}$and GTK for $p_{K^{+}}$or using 


\begin{tabular}{l|l}
\hline Process & Expected events \\
\hline$K^{+} \rightarrow \pi^{+} \pi^{0}(\gamma)$ IB & $0.064 \pm 0.007_{\text {stat }} \pm 0.006_{\text {syst }}$ \\
$K^{+} \rightarrow \mu^{+} v(\gamma)$ IB & $0.020 \pm 0.003_{\text {stat }} \pm 0.006_{\text {syst }}$ \\
$K^{+} \rightarrow \pi^{+} \pi^{+} \pi^{-}$ & $0.002 \pm 0.001_{\text {stat }} \pm 0.002_{\text {syst }}$ \\
$K^{+} \rightarrow \pi^{+} \pi^{-} e^{+} v$ & $0.013_{-0.012}^{+0.017} \pm 0.009_{\text {syst }}$ \\
$K^{+} \rightarrow \pi^{0} \mu^{+} v, K^{+} \rightarrow \pi^{0} e^{+} v$ & $<0.001$ \\
$K^{+} \rightarrow \pi^{+} \gamma \gamma$ & $<0.002$ \\
Upstream background & $\left.0.050_{-0.033}^{+0.092}\right|_{\text {stat }}$ \\
\hline Total background & $\left.0.152_{-0.033}^{+0.092}\right|_{\text {stat }} \pm 0.013_{\text {syst }}$ \\
\hline
\end{tabular}

Table 1: Summary of the background estimates summed over the two signal regions based on 2016 data sample

STRAW for $p_{\pi^{+}}$and the nominal beam parameters for $p_{K^{+}}$. The final signal acceptance, extracted from Monte-Carlo (MC) simulations, is $1 \%$ in $\mathrm{R} 1$ and $3 \%$ in $\mathrm{R} 2$.

Assuming the number of kaon decays in the fiducial volume $N_{K}=(1.21 \pm 0.02) \times 10^{11}$, the Single Event Sensitivity for a SM $K_{\pi v \bar{v}}$ decay is $S E S=\left(3.15 \pm 0.01_{\text {stat }} \pm 0.24_{\text {syst }}\right) \times 10^{-10}$. The number of kaon decays was measured from a control-triggered sample of $K^{+} \rightarrow \pi^{+} \pi^{0}$ selected using the $\pi v \bar{v}$ criteria except $\gamma$ and multiplicity rejection, and requiring $m_{m i s s}^{2}$ to be in the $\pi^{+} \pi^{0}$ region. Using the value of the SES and the branching ratio of the SM $K_{\pi v \bar{v}}$, the expected number of SM $K_{\pi v \bar{v}}$ events is $N_{K \pi v \bar{v}}=\left(0.267 \pm 0.001_{\text {stat }} \pm 0.020_{\text {sys }} \pm 0.032_{e x t}\right)$.

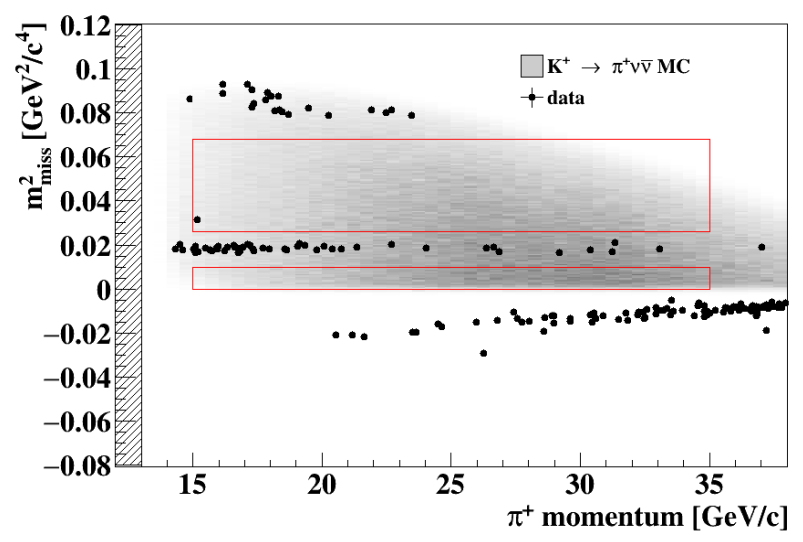

Figure 2: Reconstructed $m_{\text {miss }}^{2}$ as a function of momentum of $\pi^{+}$(markers) satisfying the $K^{+} \rightarrow \pi^{+} v \bar{v}$ selection, except the $m_{m i s s}^{2}$ and $\pi^{+}$momentum criteria. The grey area corresponds to the expected distribution of $K^{+} \rightarrow \pi^{+} v \bar{v}$ MC events. Red contours define the signal regions

The expected contributions from all background sources are shown in Table 1. Apart from $K^{+} \rightarrow \pi^{+} \pi^{-} e^{+} v$, for which the background estimation was evaluated with Monte Carlo, all other contributions were obtained using a data driven procedure validated in control regions around the two signal regions. After un-blinding the signal regions one event was found in the Region 2 as shown on Figure 2. Based on the observation of one event, the p-value for the background hypothesis is $15 \%$ and the corresponding observed upper limit is: $\mathscr{B}\left(K^{+} \rightarrow \pi^{+} v \bar{v}\right)<14 \times 10^{-10}$ 


\begin{tabular}{l|l}
\hline Process & Expected events \\
\hline$K^{+} \rightarrow \pi^{+} \pi^{0}(\gamma) \mathrm{IB}$ & $0.35 \pm 0.02_{\text {stat }} \pm 0.03_{\text {syst }}$ \\
$K^{+} \rightarrow \mu^{+} v(\gamma) \mathrm{IB}$ & $0.16 \pm 0.01_{\text {stat }} \pm 0.05_{\text {syst }}$ \\
$K^{+} \rightarrow \pi^{+} \pi^{-} e^{+} v$ & $0.22 \pm 0.08_{\text {stat }}$ \\
$K^{+} \rightarrow \pi^{+} \pi^{+} \pi^{-}$ & $0.015 \pm 0.008_{\text {stat }} \pm 0.015_{\text {syst }}$ \\
$K^{+} \rightarrow \pi^{+} \gamma \gamma$ & $0.005 \pm 0.005_{\text {syst }}$ \\
$K^{+} \rightarrow l^{+} \pi^{0} v_{l}$ & $0.012 \pm 0.012_{\text {syst }}$ \\
Upstream background & Analysis ongoing \\
\hline
\end{tabular}

Table 2: Summary of the background estimates summed over the two signal regions: preliminary estimation of expected background contributions based on 2017 data sample.

at 95\% CL. Detailed descriptions of the analysis and background estimations can be found in [10].

\subsection{Future prospects}

The result shown corresponds to the analysis of data taken in 2016, which is about $2 \%$ of the total data sample. Analysis of data collected in 2017 is ongoing with 2016-like event selection and has comparable performance. However, a number of improvements have been made in pileup treatment of IRC and SAC, that have resulted in $40 \%$ lower $\pi^{0}$ rejection inefficiency, and in usage of RICH variables. Moreover, in order to mitigate the background coming from upstream decays an additional shielding was installed in the beam line during the last part of the 2017 data taking. Later in 2018 a new final collimator was installed with the same purpose of suppressing the upstream background. The preliminary background estimations are summarized in table 2 .

\section{Searches for LNV/LFV in kaon decays}

The available data set provides the potential for precision measurements of rare kaon decays. The search for forbidden decays $K^{+} \rightarrow \pi^{-} \mu^{+} \mu^{+}$and $K^{+} \rightarrow \pi^{-} e^{+} e^{+}$was performed using data taken during three months in 2017, which corresponds to $30 \%$ of data collected by NA62 in 20162018. All the details of the analysis can be found in [11]. The reconstructed mass spectra obtained with the two analyses is shown in Figure 3. The upper limits $\mathscr{B}\left(K^{+} \rightarrow \pi^{-} e^{+} e^{+}\right)<2.2 \times 10^{-10}$ and $\mathscr{B}\left(K^{+} \rightarrow \pi^{-} \mu^{+} \mu^{+}\right)<4.2 \times 10^{-11}$ at $90 \% \mathrm{CL}$ were set. These improve on the current PDG limits by a factor of three in the electron mode and by a factor of two in the muon mode.

\section{Conclusion}

The first NA62 $K^{+} \rightarrow \pi^{+} v \bar{v}$ result based on the analysis of 2016 data [10] was reported. One event was observed in the region 2 and upper limit was set $\mathscr{B}\left(K^{+} \rightarrow \pi^{+} v \bar{v}\right)<14 \times 10^{-10}$ at $95 \%$ CL. Analysis of the 2017 data set is ongoing and preliminary background estimations were shown. Major improvements are expected with the analysis of the full data set.

New results for LNV/LFV searches in kaon decays were presented. No forbidden LNV/LFV processes have been observed and improved upper limits have been established. The updated UL at $90 \% \mathrm{CL}$ are $\mathscr{B}\left(K^{+} \rightarrow \pi^{-} e^{+} e^{+}\right)<2.2 \times 10^{-10}$ and $\mathscr{B}\left(K^{+} \rightarrow \pi^{-} \mu^{+} \mu^{+}\right)<4.2 \times 10^{-11}$. 

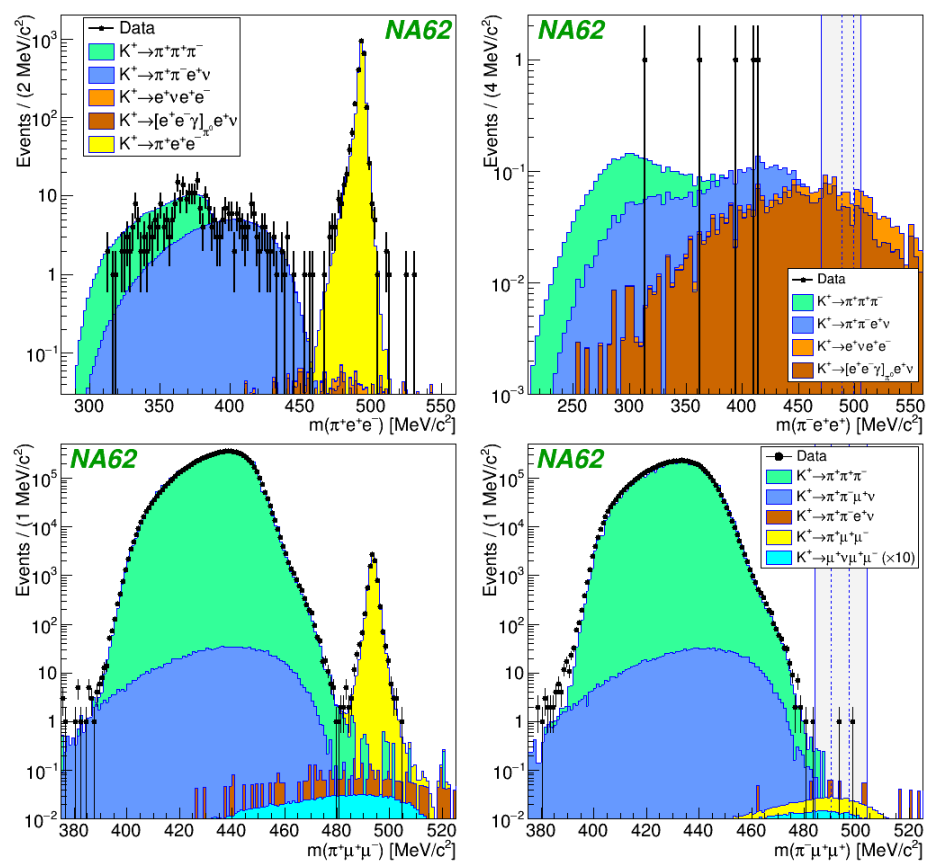

Figure 3: Illustration of the searches for lepton flavour and number violating kaon decays with the 2017 data: reconstructed mass spectra of the $\pi^{-} e^{+} e^{+}$(top right), $\pi^{-} \mu^{+} \mu^{+}$(bottom right). The signal regions and the wider regions that were blinded during the analysis are indicated with the vertical bands. Illustration of the corresponding SM decay channels $\pi^{+} e^{+} e^{-}$(top left), $\pi^{+} \mu^{+} \mu^{-}$(bottom left) final states

\section{References}

[1] A. Buras et al, JHEP 1511 (2015) 033 [arXiv: 1503.02693 [hep-ph] ]

[2] M. Blanke et al.,JHEP 0903 (2009) 108 [arXiv: 0812.3803 [hep-ph] ]

[3] T. Blažek et al., Int.J.Mod.Phys. A 29 (2014) 27 [arXiv:1410.0055 [hep-ph] ]

[4] G. Isidori et al., JHEP 0608 (2006) 064 [hep-ph / 0604074 ]

[5] A. Buras et al., JHEP 11 (2015) 166 [arXiv:1507.08672 [hep-ph] ]

[6] M. Blanke et al., Eur.Phys.J. C 76 (2016) 182 [arXiv:1507.06316 [hep-ph] ]

[7] G. Isidori et al., Eur.Phys.J. C 77 (2017) 618 [arXiv:1705.10729 [hep-ph] ]

[8] A. V. Artamonov et al. [E949 Collaboration], Phys. Rev. D 79 (2009) 092004 [arXiv: 0903.0030 [hep-ex]]

[9] E. Cortina Gil et al [NA62 Collaboration], JINST 12 (2017) P05025 [arXiv: 1703.08501 [physics.ins-det]]

[10] E. Cortina Gil et al [NA62 Collaboration], Phys. Lett. B 791 (2019) 156 [arXiv: 1811.08508 [hep-ex]]

[11] E. Cortina Gil et al. [NA62 Collaboration], submitted to Phys. Lett. B [arXiv: 1905.07770 [hep-ex]]. 